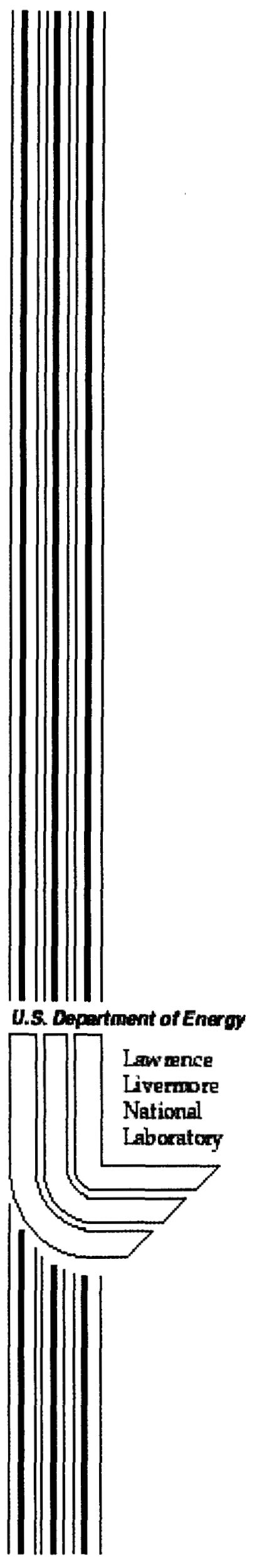

UCRL-ID-150418

\title{
Level 1 V. \& V. Test Problem 10: Escape of High Explosive Products
}

P. Dykema, S. Brandon, J. Bolstad, T. Woods, R. Klein

September 17, 2002 


\section{DISCLAIMER}

This document was prepared as an account of work sponsored by an agency of the United States Government. Neither the United States Government nor the University of California nor any of their employees, makes any warranty, express or implied, or assumes any legal liability or responsibility for the accuracy, completeness, or usefulness of any information, apparatus, product, or process disclosed, or represents that its use would not infringe privately owned rights. Reference herein to any specific commercial product, process, or service by trade name, trademark, manufacturer, or otherwise, does not necessarily constitute or imply its endorsement, recommendation, or favoring by the United States Government or the University of California. The views and opinions of authors expressed herein do not necessarily state or reflect those of the United States Government or the University of California, and shall not be used for advertising or product endorsement purposes.

This work was performed under the auspices of the U. S. Department of Energy by the University of California, Lawrence Livermore National Laboratory under Contract No. W-7405-Eng-48.

This report has been reproduced directly from the best available copy.

Available electronically at http://www.doc.gov/bridge

Available for a processing fee to U.S. Department of Energy

And its contractors in paper from

U.S. Department of Energy

Office of Scientific and Technical Information

P.O. Box 62

Oak Ridge, TN 37831-0062

Telephone: (865) 576-8401

Facsimile: (865) 576-5728

E-mail: reports@adonis.osti.gov

Available for the sale to the public from

U.S. Department of Commerce

National Technical Information Service

5285 Port Royal Road

Springfield, VA 22161

Telephone: (800) 553-6847

Facsimile: (703) 605-6900

E-mail: orders@ntis.fedworld.gov

Online ordering: http://www.ntis.gov/ordering.htm

OR

Lawrence Livermore National Laboratory

Technical Information Department's Digital Library

http://www.llnl.gov/tid/Library.html 


\title{
Level 1 V. \& V. Test Problem 10: Escape of High Explosive products
}

\author{
Pieter Dykema, Scott Brandon, John Bolstad, Todd Woods \\ and Richard Klein \\ A-Program V\&V Program \\ Lawrence Livermore National Laboratory
}

September 17, 2002

\section{Introduction}

The Escape of High Explosive (HE) Products test is a hydrodynamics only problem designed to test the codes' ability to model the propagation of an unsupported, HE detonation wave. An ideal gas is used to model the HE. The detonation products expand to the right and interact at the left with a constant velocity piston. The choice of $\gamma=3.0$ and a careful selection of the piston velocity produces straight-line characteristics and makes possible the calculation of the exact solution. This problem tests the calculation of flow near a cavitation front where large gradients exist and is particularly difficult because both sets of characteristics become coincident at the front.

Multidimensional Lagrange/ALE codes allow flexibility in constructing the computational mesh. For 2 and 3-D calculations, the ability of the code to maintain symmetry can also be tested. Eulerian codes may have difficulty implementing the constant velocity piston (perhaps an actual piston could be modeled).

Many codes use a "programmed burn" model to represent the HE detonation wave. In this model, the velocity of the detonation front is precomputed. For these codes, only the selfconsistent hydrodynamics occurring behind the detonation front is actually tested.

Finally, practical problems are seldom 1-D. Modeling HE detonations in multiple dimensions introduces many more interactions and other possibilities than can be tested by this 1-D problem.

\section{Problem Description and Setup}

This is a one-dimensional planar, single-fluid problem using an ideal gas to model an unsupported high explosive (HE) detonation. The detonation products expand to the right into a vacuum and interact at the left with a constant-velocity piston. This problem tests the calculation of the flow near a cavitation front where large gradients exist. The calculation is particularly difficult because both sets of characteristics become coincident at the cavitation front. The choice of $\gamma=3.0$ 
produces straight-line characteristics which makes possible the calculation of the exact solution. A more complete description of the problem is found in Reference 1.

The HE is Fickett and Rivard Comp B with an initial density $\rho=1.6 \mathrm{gm} / \mathrm{cc}$. At $t=0$ the $\mathrm{HE}$ will be detonated at the left boundary with a detonation velocity $D_{C J}=8.5 \times 10^{5} \mathrm{~cm} / \mathrm{sec}$ and the HE product equation of state is $p=(27 / 256)\left(D_{C J}{ }^{2} / \rho_{0}^{2}\right) \rho^{3}$. The region will be driven about $30 \mathrm{~cm}$ to the right. Thus, Lagrangian codes should provide a "free" boundary condition; Eulerian codes need to provide about $50 \mathrm{~cm}$ of "vacuum" on the right. The left boundary of the problem is driven to the right at a constant velocity of $0.5 \times 10^{5} \mathrm{~cm} / \mathrm{sec}$ (Eulerian codes could, perhaps, use a very massive actual piston).

Compute on a 3D "box" initially $10 \mathrm{~cm}$ long with reflecting boundaries in the transverse direction. Compute until time $t=70 \mu \mathrm{s}$ is reached. Do four problems using an initially uniform grid of $100,200,400$, and 800 zones.

As output, in addition to the comparisons to quantities provided in Fickett and Rivard Table 6.2 (Note: the time values must be multiplied by 10 ), supply values of the density, pressure and velocity vs. radius at the final time. For each quantity, produce plots superimposing the calculational result for all resolutions and the analytic solution. Include a time history plot of the total energy in the problem. Plot the log of the $\ell_{1}$ norm of the difference between the calculated and analytical solution of density as a function of the inverse of the initial grid resolution. The $\ell_{1}$ norm is calculated as follows:

$$
\ell_{1}=\sum_{i=1}^{\text {nzones }}\left|\operatorname{Simulation}\left(x_{i}\right)-\operatorname{Analytic}\left(x_{i}\right)\right| \triangle x_{i}
$$

\section{Analytic Solution}

The following is a brief review of the simplest practical model of high explosive (HE) detonations and a derivation of the solution to the equations of motion that comprises the test problem. The model is a one dimensional front moving with the Chapman-Jouguet $(\mathrm{CJ})$ velocity with respect to the unburned HE. The burn chemistry is assumed to be completed instantaneously upon arrival of the detonation front. The equation of state of the reaction products is modeled as an ideal gas with constant heat capacity. A particular choice of the polytropic index allows a simple solution based on the method of characteristics.

\subsection{Derivation of the Analytic Model}

The HE detonation front is modeled as a planar discontinuity that is in steady state in its own rest frame. In the rest frame of the unburned material the Rankine-Hugoniot jump conditions for mass and momentum conservation are

$$
\begin{gathered}
\rho_{0} D=\rho(D-u) \\
p-p_{0}=\rho_{0} u D
\end{gathered}
$$


where $\rho_{0}$ and $p_{0}$ are the mass density and pressure of the HE prior to detonation, $\rho$ and $p$ are post-detonation values, $u$ is the velocity of the detonation products and $D$ is the velocity of the burn front. Introducing the specific volume

$$
v=\frac{1}{\rho}
$$

and eliminating $u$ between (1) and (2) results in the so-called "Rayleigh line,"

$$
p-p_{0}+\rho_{0}^{2} D^{2}\left(v-v_{0}\right)=0
$$

The third jump condition, expressing conservation of energy across the detonation front, can be written as the "Hugoniot curve,"

$$
E-E_{0}=\frac{1}{2}\left(p+p_{0}\right)\left(v_{0}-v\right)
$$

where $E_{0}$ and $E$ are the specific $H E$ energies in front of and behind the detonation wave. Given an equation of state for $E$ and a detonation velocity $D$, the post-detonation state $(p, v)$ is obtained from the pre-detonation state as the intersection of the Hugoniot curve (4) with the Rayleigh line (3).

The test problem assumes a very simple chemical reaction model of the detonation process. No chemical kinetics equations are actually solved. Rather, a state dependent equilibrium model is used, according to which the chemical composition of the HE is determined by the instantaneous state of the system. We have

$$
E=E(p, v, \lambda)
$$

where $\lambda$ is a parameter governing the degree of chemical reaction such that $\lambda=0$ corresponds to the initial, unburned state and $\lambda=1$ represents the detonation products. That is,

$$
E=E(p, v, \lambda=1)
$$

and

$$
E_{0}=E\left(p_{o}, v_{0}, \lambda=0\right)
$$

in (4). Because of the approximation of zero thickness for the chemical reaction region the transition from $\lambda=0$ to $\lambda=1$ is instantaneous.

The equation of state is taken to be an ideal gas with constant heat capacity,

$$
E=c_{v} T-\lambda q=\frac{p v}{\gamma-1}-\lambda q
$$

where $\gamma$ is the constant polytropic index and $q \geq 0$ is the constant heat of complete reaction. Substituting (6) into (4) yields the ideal gas Hugoniot curve, the locus of all possible final states for any detonation: 


$$
\left(\frac{p}{p_{0}}+\mu^{2}\right)\left(\frac{v}{v_{0}}-\mu^{2}\right)=1-\mu^{4}+2 \mu^{2} \frac{q}{p_{0} v_{0}}
$$

where

$$
\mu^{2} \equiv \frac{\gamma-1}{\gamma+1}
$$

For positive $q$ this curve does not run through the initial state. The implication of this is that, unlike an ordinary shock, there exists a range of values of $D^{2}$ satisfying

$$
D^{2} \geq \frac{\gamma p_{0}}{\rho_{0}}
$$

for which there is no real solution to the simultaneous equations (3) and (7). Solving this system for the inverse of the compression gives

$$
\frac{v}{v_{0}}=\frac{1}{\beta} \frac{\gamma(1+\beta) \pm \beta r^{\frac{1}{2}}}{\gamma+1}
$$

where

$$
\beta \equiv \frac{\rho_{0} D^{2}}{p_{0}}
$$

and

$$
r \equiv\left(1-\frac{\gamma}{\beta}\right)^{2}-2\left(\gamma^{2}-1\right) \frac{q}{D^{2}}
$$

The specific volume ratio (9) is complex and thus nonphysical for $r<0$. This occurs for a given $q$ when $D^{2}$ is sufficiently small. The single root at $r=0$ defines the Chapman-Jouguet point, characterized by the $\mathrm{CJ}$ detonation velocity

$$
D^{2}=\frac{\gamma p_{0}}{\rho_{0}}+\left(\gamma^{2}-1\right) q+\left[\left(\gamma^{2}-1\right)^{2} q^{2}+2\left(\gamma^{2}-1\right) q \frac{\gamma p_{0}}{\rho_{0}}\right]^{\frac{1}{2}}
$$

the minimum detonation velocity possible in the model. Values of $D^{2}$ greater than the $\mathrm{CJ}$ value yield positive values of $r$ and from (9) two possible final states for the detonation products. The "+" root is the so-called "weak" solution, characterized by final state compression and pressure that are less than the $C J$ values. The "." root yields the "strong" solution wherein the reaction products have a higher compression ratio and pressure than the $\mathrm{CJ}$ result. It is straightforward to show that the strong solution is unlikely to obtain. This is because the reaction product fluid velocity for this solution is subsonic in the shock rest frame, rendering the detonation front subject to dissipation by a following rarefaction. In fact, however, unsupported detonations appear quickly to reach a steady velocity and thereafter to be self-sustaining. Thus, from (3) and (9),

$$
\frac{p}{p_{0}}=\frac{1+\beta \mp \beta r^{\frac{1}{2}}}{\gamma+1}
$$


The isentropic sound speed behind the front follows as

$$
c_{s}^{2}=\frac{\gamma p}{\rho}=\left[\frac{\gamma\left(1+\frac{1}{\beta}\right) D}{\gamma+1}\right]^{2} R_{\mp}
$$

where

$$
R_{\mp} \equiv 1 \mp \frac{1-\frac{1}{\gamma}}{1+\frac{1}{\beta}} r^{\frac{1}{2}}-\frac{r}{\gamma\left(1+\frac{1}{\beta}\right)^{2}}
$$

The post-detonation fluid velocity is given by (1),

$$
u=D\left(1-\frac{v}{v_{0}}\right)=D\left[1-\frac{\gamma\left(1+\frac{1}{\beta}\right)}{\gamma+1} \mp \frac{r^{\frac{1}{2}}}{\gamma+1}\right]
$$

so that

$$
u+c_{s}=D\left[1+\frac{\gamma\left(1+\frac{1}{\beta}\right)}{\gamma+1}\left(R_{\mp}^{\frac{1}{2}}-1\right) \mp \frac{r^{\frac{1}{2}}}{\gamma+1}\right]
$$

The upper sign in (9) through (17) corresponds to the weak solution, the lower sign to the strong. It follows by inspection of (17) that because

$$
R_{-}^{\frac{1}{2}}-1<0
$$

the weak solution satisfies

$$
u+c_{s}<D
$$

indicating that the flow behind the shock is supersonic with respect to the shock. Similarly, because of the inequality

$$
\gamma\left(1+\frac{1}{\beta}\right)\left(R_{+}^{\frac{1}{2}}-1\right)+r^{\frac{1}{2}}>0
$$

the strong solution is characterized by

$$
u+c_{s}>D
$$

and the following flow is subsonic. The $\mathrm{CJ}$ state is that solution for which $r=0$ and $R=1$ so that, from (17),

$$
u+c_{s}=D
$$

and the fluid moves away from the burn front at precisely the post-detonation sound speed. 
Although there is some evidence to support the conclusion that planar detonations can satisfy weak solutions, our test problem assumes that the final state is, in fact, the $\mathrm{CJ}$ state. Collecting the results for this case we have, in addition to expression (12) for the detonation velocity in terms of the heat of reaction, the final state values (9) and (13) for $v$ and $p$.

$$
\frac{v}{v_{0}}=\frac{\gamma}{\gamma+1}\left(1+\frac{p_{0}}{\rho_{0} D^{2}}\right)
$$

and

$$
p=\frac{1}{\gamma+1}\left(p_{0}+\rho_{0} D^{2}\right)
$$

The post-detonation sound and fluid velocities follow from (14) and (16);

$$
c_{s}=\frac{\gamma}{\gamma+1} D\left(1+\frac{p_{0}}{\rho_{0} D^{2}}\right)
$$

and

$$
u=\frac{D}{\gamma+1}\left(1-\gamma \frac{p_{0}}{\rho_{0} D^{2}}\right)
$$

The final simplification we will make in the test problem is the constraint that the pressure of the reactants prior to detonation be negligible by comparison with $\rho_{0} D^{2}$. Setting $p_{0}=0$ in (21) through (24) allows the simplification that the post-detonation state of the reaction products depends only on $\gamma, \rho_{0}$ and the CJ velocity (12),

$$
D^{2}=2\left(\gamma^{2}-1\right) q
$$

In our test problem the detonation products will expand forward into a vacuum. The approximation of zero pressure in front of the detonation wave implies that the sound speed in this region is also zero, so the wave trajectory is not influenced by the HE-vacuum boundary prior to arrival at that boundary.

\subsection{The Solution by the Method of Characteristics}

In the characteristic analysis to follow we will find it convenient to solve directly for the sound speed in various regions of the isentropic domain behind the detonation front. Throughout this domain the reaction products will satisfy a pressure density relationship of the form

$$
p=k \rho^{\gamma}
$$

The constant $k$ is determined by the requirement that this relation hold for the $\mathrm{CJ}$ pressure (22) and density (21). Thus,

$$
k=\left(\frac{\gamma}{\gamma+1}\right)^{\gamma} \frac{1}{\gamma+1} \rho_{0}^{1-\gamma} D^{2}
$$


Using

$$
c_{s}^{2}=\frac{\gamma p}{\rho}
$$

and eliminating $p$ gives an expression for the density in the post-detonation region in terms of the local sound speed,

$$
\frac{\rho}{\rho_{0}}=\left(\frac{\gamma+1}{\gamma}\right)^{\frac{\gamma+1}{\gamma-1}}\left(\frac{c_{s}}{D}\right)^{\frac{2}{\gamma-1}}
$$

This result in turn, together with (26), gives the pressure throughout the domain,

$$
p=\left(\frac{\gamma+1}{\gamma}\right)^{\frac{2 \gamma}{\gamma-1}} \frac{1}{\gamma+1} \rho_{0} D^{2}\left(\frac{c_{s}}{D}\right)^{\frac{2 \gamma}{\gamma-1}}
$$

A characteristic analysis of the Euler equations in one spatial dimension yields three real eigenvalues, which are the slopes of the characteristic curves in the (space, time) $=(x, t)$ plane. The slope of the so- called $C^{0}$ characteristic is the fluid velocity itself,

$$
\xi^{0}=u
$$

The $C^{ \pm}$characteristics have slopes

$$
\xi^{ \pm}=u \pm c_{s}
$$

The fluid specific entropy is constant along each $C^{0}$ curve. In what follows, however, we will focus more on the $C^{ \pm}$families of curves. If $\eta$ is an affine parameterization of one of these curves,

$$
\frac{d}{d \eta} \propto \partial_{t}+\left[u(\eta) \pm c_{s}(\eta)\right] \partial_{x}
$$

we have the result that

$$
\frac{1}{\rho c_{s}} \frac{d p}{d \eta} \pm \frac{d u}{d \eta}=0
$$

with the sign depending on which type of curve it is. From (27) and (28),

$$
\frac{1}{\rho c_{s}} \frac{d p}{d \eta}=\frac{2}{\gamma-1} \frac{d c_{s}}{d \eta}
$$

Equation (32) can be therefore be integrated immediately, with the result that

$$
\frac{2}{\gamma-1}\left[c(\eta)-c\left(\eta_{0}\right)\right] \pm\left[u(\eta)-u\left(\eta_{0}\right)\right]=0
$$

where $\eta$ and $\eta_{0}$ are any two points on the curve. Evidently, the value 


$$
\gamma=3
$$

of the adiabatic index gives, from (34),

$$
c(\eta) \pm u(\eta)=c\left(\eta_{0}\right) \pm u\left(\eta_{0}\right)
$$

That is, the values of the slopes of the $C^{ \pm}$characteristics are constants; they are straight lines in the $(x, t)$ plane. This is an important simplification that makes possible an analytic solution to the test problem and for this reason we stipulate that $\gamma=3$.

The geometry of the test problem is a linear region of initially undetonated HE bounded on the right by vacuum and on the left by a piston moving with specified constant velocity $u_{p}$. The detonation is initiated at time $t=0$ at $x=0$, the left boundary, and moves to the right with specified velocity $D$. The piston is also started at $t=0$. Fig. 1 summarizes the problem. Also shown in Fig. 1 are five trajectories, labeled " $A$ " through " $E$," that divide the space-time solution domain into five regions, labeled "l" through " $V$." We will consider in turn the five curves and then the five regions.

The Characteristic Solution

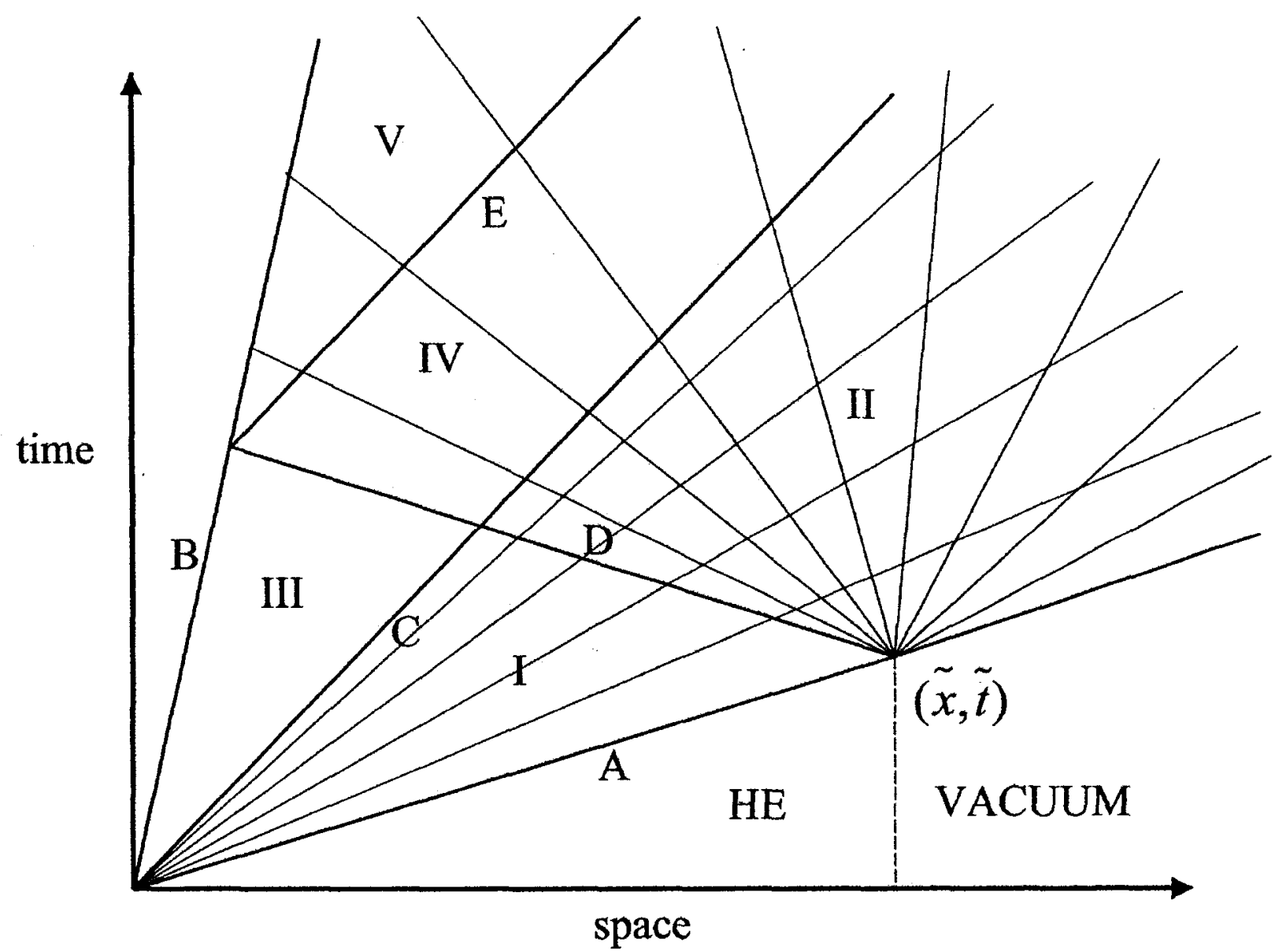

Figure 1: Graphical representation of the characteristic solution 
Shown on Fig. 1 are domains I - V within each of which the solution takes a simple analytic form. Also shown are the bounding curves $A-E$ of the domains and two characteristic fans discussed in the text.

Curve $A$ is the detonation front trajectory,

$$
x=D t
$$

Let $\tilde{x}$ be the initial length of the HE. Along curve A from the origin to event $(x, t)=\left(\tilde{x}, \tilde{t}=\frac{\tilde{x}}{D}\right)$ the sound speed and fluid velocity take their CJ values. From (23) and (24),

$$
c_{s}=\frac{3}{4} D
$$

and

$$
u=\frac{1}{4} D
$$

Since $u+c_{s}=D$, the detonation front is its own $C^{+}$characteristic. The $C^{-}$characteristic from any point on the curve has (inverse) slope

$$
u-c_{s}=-\frac{1}{2} D
$$

The characteristic net collapses at $(\tilde{x}, \tilde{t})$ where the detonation front first encounters the influence of the vacuum boundary. From (2) the pressure $p$ and so the sound speed drop discontinuously to zero with the result that all three characteristics emanating from events on $A$ coincide with $A$ :

$$
u+c_{s}=u=u-c_{s}=D
$$

From this event outward the detonation front is causally disconnected from the rest of the evolution. The collapse of the net at the discontinuity yields a $C^{-}$fan at $(\tilde{x}, \tilde{t})$ that provides knowledge of the HE-vacuum boundary to regions II, IV and V.

Curve $B$ is the piston trajectory,

$$
x=u_{p} t
$$

As a boundary, the piston provides the condition that the fluid velocity along its trajectory is

$$
u=u_{p}
$$

Curve $C$ is the leading $C^{+}$piston characteristic; that is, the one emanating from the origin. The equation of this curve follows from applying (43) and (40) at the origin. Thus, solving for the sound speed,

$$
c_{s}=u_{p}+\frac{1}{2} D
$$


so that

$$
u+c_{s}=2 u_{p}+\frac{1}{2} D
$$

It follows that curve $C$ is described by

$$
x=\left(2 u_{p}+\frac{1}{2} D\right) t
$$

Because curves $\mathrm{A}$ and $\mathrm{C}$ representing $C^{+}$characteristics from the origin are not identical, there is a $C^{+}$fan emanating from the origin that is bounded by these curves. This fan provides knowledge of the initial position of the piston to regions I and II.

Curve $\mathrm{D}$ can be considered to be either the last $C^{-}$characteristic from the detonation front before it arrives at the end of the detonation region or the first characteristic in the $C^{-}$fan at $(\tilde{x}, \tilde{t})$. It separates regions I and III from II, IV and V. It must have the generic form

$$
x-\tilde{x}=\left(u-c_{s}\right)(t-\tilde{t})
$$

The value of the invariant $u-c_{s}$ is the $C J$ value, given by (40), so that curve $D$ is given by

$$
x=\frac{1}{2}(3 \tilde{x}-D t)
$$

Curve $\mathrm{E}$ is the piston $C^{+}$characteristic from the event that is the intersection of the piston path with curve $\mathrm{D}$. At an event $(x, t)=\left(x_{p}, t_{p}\right)=\left(u_{p} t_{p}, t_{p}\right)$ on the piston path the $C+$ characteristic must have the form

$$
x-x_{p}=\left(u+c_{s}\right)\left(t-t_{p}\right)
$$

Evaluating expressions (42) and (48) for curves B and D at $\left(x_{p}, t_{p}\right)$ results in

$$
t_{p}=\frac{3}{2} \frac{\tilde{x}}{u_{p}+\frac{1}{2} D}
$$

Following the analysis of curve $C$, the invariant $u+c_{s}$ is given by (45). Substituting these results into (49) yields the equation for curve $E$ :

$$
x=-\frac{3}{2} \tilde{x}+\left(2 u_{p}+\frac{1}{2} D\right) t
$$

Region $I$ is bounded by curves $C$ and $D$ and by the segment of the detonation trajectory $A$ between the origin and the vacuum. The $C^{+}$characteristics come from the fan generated by the discontinuity at the origin:

$$
x=\left(u+c_{s}\right) t
$$


The $C^{-}$characteristics come from the detonation curve $\mathrm{A}$ and provide the value of the Riemann invariant $u-c_{s}$ given by the $C J$ value (40). Solving these equations for the sound speed and the fluid velocity gives for the space-time dependencies of these quantities in region I the results

$$
c_{s}=\frac{1}{2}\left(\frac{x}{t}+\frac{1}{2} D\right)
$$

and

$$
u=\frac{1}{2}\left(\frac{x}{t}-\frac{1}{2} D\right)
$$

The density and pressure in region I follow from (27) and (28) with $\gamma=3$;

$$
\rho=\frac{16}{9} \rho_{0} \frac{c_{s}}{D}
$$

and

$$
p=\frac{16}{27} \rho_{0} D^{2}\left(\frac{c_{s}}{D}\right)^{3}
$$

Applied to region I these equations describe a rarefaction following the initial compression of the $\mathrm{HE}$ detonation products in which the density on a given time slice falls off linearly in space.

In region II the $C^{+}$characteristics are the same as in region I, the fan generated at the origin. The $C^{-}$characteristics are the fan generated at $(\tilde{x}, \tilde{t})$. The $C^{+}$curves are described by (52) while the $C^{-}$equations come from (47). Solving these equations for the sound speed and the fluid velocity,

$$
c_{s}=\frac{1}{2}\left(\frac{x}{t}-\frac{x-\tilde{x}}{t-\tilde{t}}\right)
$$

and

$$
u=\frac{1}{2}\left(\frac{x}{t}+\frac{x-\tilde{x}}{t-\tilde{t}}\right)
$$

The density and pressure follow from (55) and (56). Region II describes the density and pressure reductions in the $\mathrm{HE}$ burn product as it expands into the vacuum region and the influence of this expansion as knowledge of the vacuum boundary works its way back to the left toward the leading $C^{+}$characteristic of the piston.

The $C^{+}$characteristics in region III come from the piston. The $C^{-}$characteristics are from curve $\mathrm{A}$, the detonation front, and bring the information that the invariant $u-c_{s}$ satisfies (40) throughout III. In particular, (40) must be satisfied on the piston itself, where we know also that the fluid velocity is the same as the piston velocity. Thus, $(40)$ can be solved for the sound speed at the piston. The result is equation (44). To this in turn we add the piston fluid velocity and obtain

$$
u+c_{s}=2 u_{p}+\frac{1}{2} D
$$


for the $C^{+}$invariant throughout region III. This is an extension to all of region III of the result (45) for the bounding curve $C$. Equations (40) and (59) can now be solved for $c_{s}$ and $u$ with the results that throughout region III

$$
c_{s}=u_{p}+\frac{1}{2} D
$$

and

$$
u=u_{p}
$$

From (55) and (56), the constancy of $c_{s}$ implies that the density and pressure are also constant in region III. As time elapses this constant region moves out ahead of the piston behind curve $C$ until the rarefaction curve $D$ is encountered, whereupon region III retreats back to the piston.

The $C^{+}$characteristics in region IV are the same congruence as in region III; they come from the piston and bring the Riemann invariant (59). The $C^{-}$curves are the fan from $(\tilde{x}, \tilde{t})$, as in region II, with form (47). Thus, in region IV we have

$$
c_{s}=u_{p}+\frac{D}{2}\left(\frac{1}{2}-\frac{x-\tilde{x}}{D t-\tilde{x}}\right)
$$

and

$$
u=u_{p}+\frac{D}{2}\left(\frac{1}{2}+\frac{x-\tilde{x}}{D t-\tilde{x}}\right)
$$

Like region I, region IV is a rarefaction.

The final stage of the evolution of the test problem is contained in region $\mathrm{V}$. In $\mathrm{V}$, the inwardly propagating rarefaction from the vacuum boundary has reached the piston and has reflected off it as curve $E$. The fluid velocity along the segment of curve $B$ bounding region $V$ is still the piston velocity itself, but the sound speed along this segment is no longer the region III constant value given by $(60)$. As in regions II and IV the $C^{-}$characteristics come from the fan generated at $(\tilde{x}, \tilde{t})$ and are described by (47). The intersection of one of these curves with the piston curve $\mathrm{B}$ at an event $\left(x_{p}, t_{p}\right)$, where the fluid velocity is $u_{p}$, yields the sound speed at the piston as a function of position along it:

$$
c_{s}\left(t_{p}\right)=\left(D-u_{p}\right) \frac{\tilde{t}}{t_{p}-\tilde{t}}
$$

The $C^{+}$characteristics come from the piston and have the form (49). Because $u+c_{s}$ is constant along a $C^{+}$curve, we can substitute $u_{p}+c_{s}\left(t_{p}\right)$ into (49) and solve for $t_{p}$ :

$$
t_{p}=\tilde{t} \frac{x-u_{p} t+\left(D-u_{p}\right) t}{x-u_{p} t+\left(D-u_{p}\right) \tilde{t}}
$$

Given an arbitrary event $(x, t)$ in region $\mathrm{V},(65)$ gives the time at which the $C^{+}$characteristic at that event intersects the piston world line B. Using (65) in (64), adding $u_{p}$ and equating the result to $u+c_{S}$ gives an expression valid throughout the region: 


$$
u+c_{s}=\frac{x+\left(D-2 u_{p}\right) \tilde{t}}{t-\tilde{t}}
$$

Finally, (66) and (47) are two equations to be solved for the sound speed and fluid velocity in region $\mathrm{V}$ :

$$
c_{s}=\left(D-u_{p}\right) \frac{\tilde{t}}{t-\tilde{t}}
$$

and

$$
u=\frac{x-u_{p} \tilde{t}}{t-\tilde{t}}
$$

The density and pressure in region $V$ are obtained by using the sound speed result (67) in (55) and (56). Both fields are constant on a given time slice.

\subsection{Discussion of the Analytic Solution}

Curves of density vs. $x$ from the analytical solution for several thematic times during the evolution are shown on Fig. 2. The initial HE rod is $10 \mathrm{~cm}$ long with a density of $1.6 \mathrm{~g} / \mathrm{cm}^{3}$. The detonation velocity is $0.0085 \mathrm{~cm} /$ shake and the piston velocity is $0.0005 \mathrm{~cm} / \mathrm{shake}$. The detonation front breakout time is then 1176.47 shakes and, from Section 3, the region III density behind the front is $1.5895 \mathrm{~g} / \mathrm{cm}^{3}$. Curves $A$ and $B$, at 325 and 750 shakes, show the detonation wave as it propagates through the medium. The initial compression comes from the CG jump conditions, giving a compression of $4 / 3$ and taking the density to a value of $2.13333 \mathrm{~g} / \mathrm{cm}^{3}$. This is followed by the region I linear ramp down to the final region III density value. Referring to Fig. 1, curves $\mathrm{A}$ and $\mathrm{B}$ are time slices through regions $\mathrm{III}$ and $\mathrm{I}$.

Curve $C$ in Fig. 2 represents the density profile at a time of 1250 shakes, just after the breakout of the detonation front into vacuum. The density at the leading edge of the solution drops to zero. This density behavior is maintained for the duration of the subsequent evolution. The peak value of the compression drops to the region II/region I (curve D in Fig. 1) intersection value. Again there follows the region I ramp to the constant region III density value. In Fig. 1 curve $C$ is a time slice through regions III, $I$, and II.

Curve $\mathrm{D}$ in Fig. 2, taken at $t=2000$ shakes, is an example of a time slice through region III, IV, and II as depicted in Fig. 1. There is no longer a compressed region relative to the initial density. Rather, a rarefaction is eating its way back in toward the piston.

Finally, curves $\mathrm{E}$ and $\mathrm{F}$ in Fig. 2 for $t=5000$ shakes and $t=7000$ shakes, are time slices through the final phase of the evolution, intersecting the Fig. 1 regions V, IV, and II. The reflected rarefaction in region $V$ is characterized by constant density and pressure on a given time slice, with these values decreasing as a function of time. The piecewise linearity of the density profile is a striking feature of the analytic solution. 


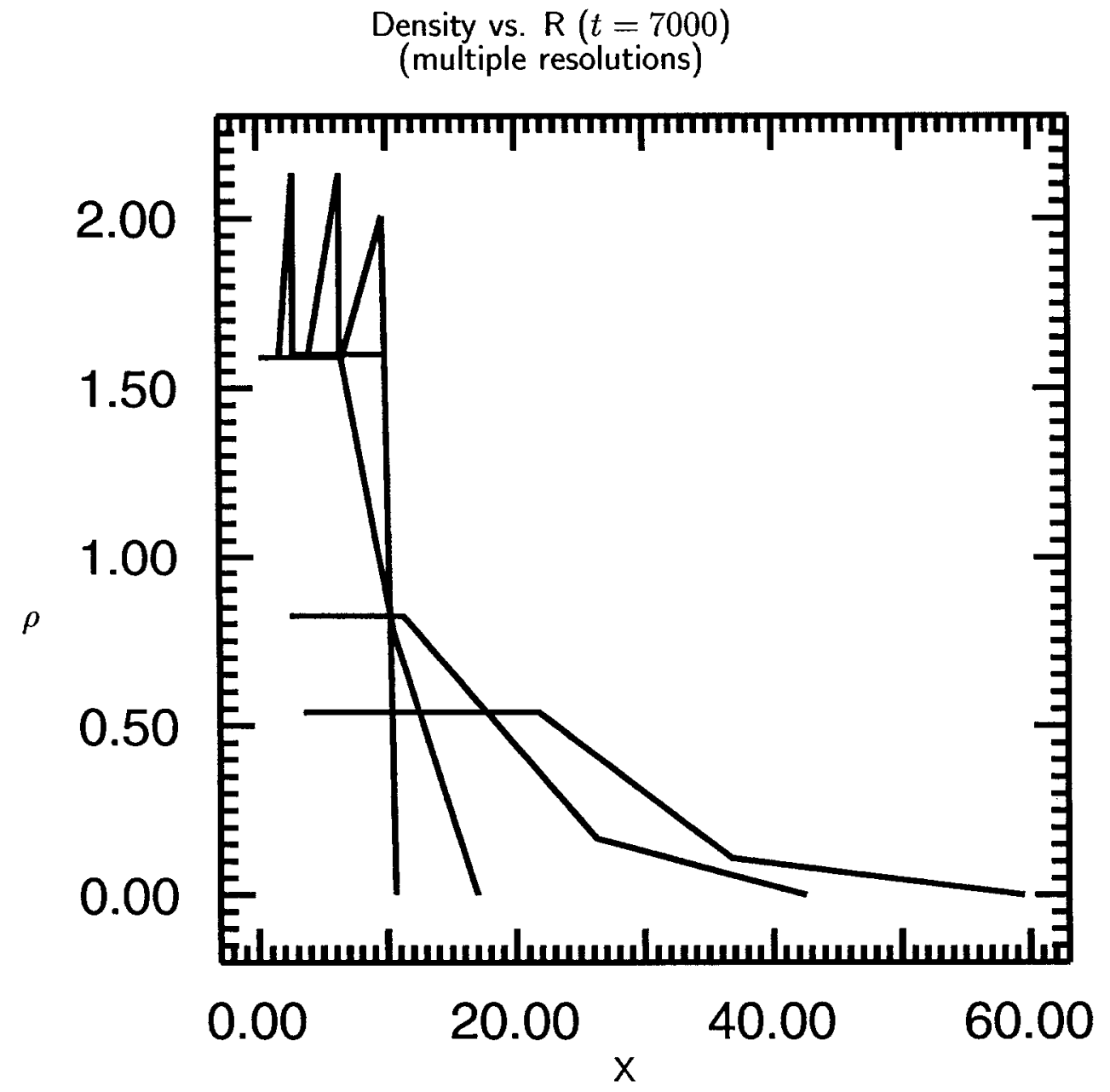

A density at $t=325$ shakes (red)

B density at $t=750$ shakes (magenta)

$C$ density at $t=1250$ shakes (green)

D density at $t=2000$ shakes (cyan)

$\mathrm{E}$ density at $t=5000$ shakes (blue)

$\mathrm{F}$ density at $t=7000$ shakes (black)

Figure 2: Density $(\rho)$ plots from the analytical solution for multiple times 


\section{Numerical Results}

The Escape of HE Products problem tests the code's ability to model an unsupported high explosive detonation in planar geometry. The choice of $\gamma=3.0$ for the HE material produces straight-line characteristics which makes possible the evaluation of the exact solution. The calculation is particularly difficult near the cavitation front where both sets of characteristics become coincident and large gradients exist.

Many codes, including CALE, use a "programmed burn" approximation to model the HE detonation. In this model, the position of the HE burn front is precalculated and therefore constrained to move at the prescribed velocity. The width of the HE burn front, like a hydrodynamic shock, is seldom resolved by the problem zoning. Additional approximations are required to spread the burn front in both time and space in order to resolve the flow. For programmed burn models, the ability of the codes to match the analytic solution behind the detonation is measured.

The Escape of HE Products test is a 1-D planar problem which can be calculated using 1, 2, or 3-D implementations. Mesh convergence studies for 3 times (750, 2000, and 7000) using an initially uniform mesh are utilized to more completely measure the code's ability to model this HE detonation wave.

\subsection{CALE Results}

CALE is a 2-D code. Cartesian geometry with 1 zone in the transverse direction is used to model the HE detonation. The energy deposition rate from the propagating HE burn blast wave is specified by the programmed burn HE model. After the burn wave has passed a given zone and all of the HE energy has been deposited, the resulting flow is self-consistently calculated. One HE burn model parameter, fhe, which modifies the width of the simulated detonation wave, had to be doubled to obtain reasonable results for mesh resolutions greater than 100 zones.

\subsubsection{CALE Lagrangian Calculations}

Curves of density vs, $x$ from the CALE code for resolutions of $25,50,100,200,400,800$, 1600,3200 and 6400 zones are shown on Fig. 3 taken at time $t=750$. The sharp increase in density at about $6.3 \mathrm{~cm}$ is produced by the front of the $\mathrm{HE}$ detonation wave. Behind the detonation front, the density falls linearly. The interaction with the piston keeps the density of the burned $\mathrm{HE}$ at very nearly the same as the initial density of the unburned HE. The convergence of the CALE calculations as mesh resolution is increased (especially evident near the corners of the HE burn wave) to the analytical solution is shown. The largest discrepancy between the calculated densities and the analytical solution occurs at the left boundary of the problem (piston interface). Here the density is decreased (temperature/energy is correspondingly increased) by a "wall" effect similar to that seen in the Noh test problems. Although the $\Delta x$ extent of the boundary interaction appears to diminish with increasing mesh resolution, the magnitude of the disturbance remains constant.

Fig. 4 shows the same data as Fig. 3, but with the plot limits zoomed to show details of the HE burn region. The density curve produced by the lowest resolution calculation (the 25 zone or red curve) shows significant deviations from the values of all higher resolutions. The calculations also fail to reproduce the density peak located at the front of the compression wave. 
Density vs. $\mathrm{R}(t=7000)$

(multiple resolutions)

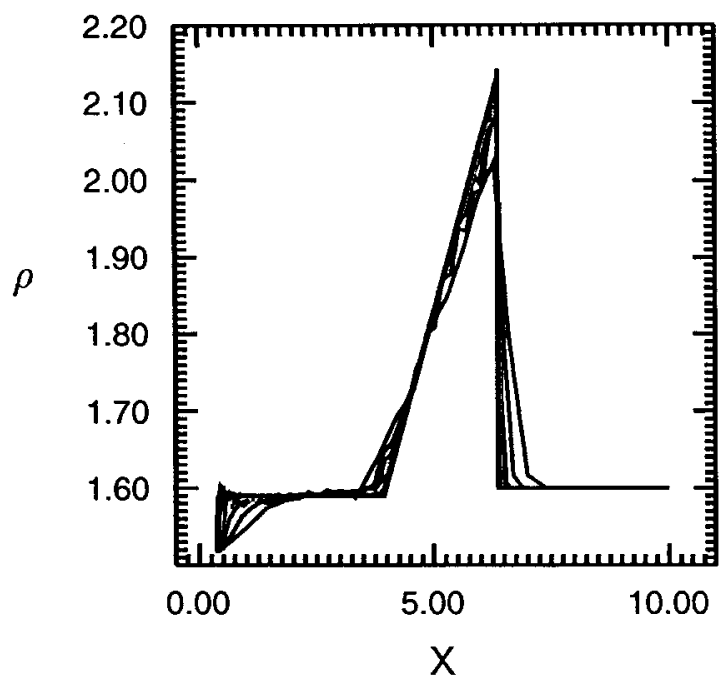

A - I density $[25,50,100,200, \quad, 800$,
$1600,3200 \& 6400$ zones]
J density [analytic solution]

Figure 3: Density $(\rho)$ plots from CALE calculations at $t=750$ for multiple resolutions
Density vs. $R(t=750)$

(multiple resolutions [zoomed])

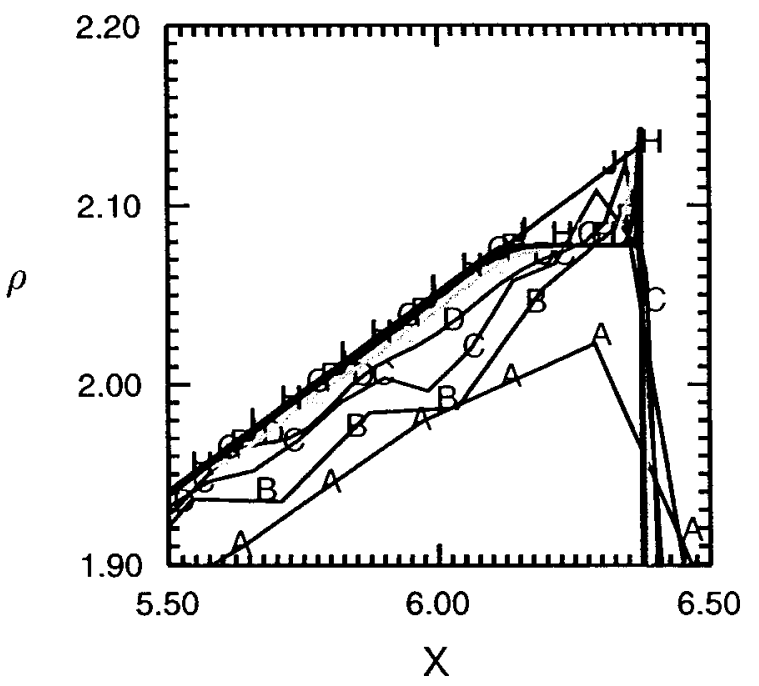

A - I density $[25,50,100,200,800$,
$1600,3200 \& 6400$ zones $]$

$\mathrm{J}$ density [analytic solution]

Figure 4: Density $(\rho)$ plots from CALE calculations at $t=750$ for multiple resolutions [zoomed]

As the resolution increases, the calculations produce a density spike (which reaches higher values than the analytical solution) followed by a flat region. After this density spike, the calculational density remains constant until it intersects the linear decreasing portion of the analytical solution. Perhaps this behavior is caused by the necessity of artificially increasing the width of the HE detonation front to contain a few zones. The convergence of the calculational values of density to that of the analytical solution is most easily seen near the density peak and at the tail of the detonation wave. At other locations, all of the calculational curves more closely match the analytical solution. The lower resolution (50 - 200 zone calculations colored from red to green) density curves present an "accordion" like folding in density space. However, these artifacts decay as the resolution increases.

Curves of density vs. $x$ from the CALE code for resolutions of $25,50,100,200,400,800$, 1600,3200 and 6400 zones are shown on Fig. 5 taken at time $t=2000$. The HE detonation has completed the burn of all available HE (initially $10 \mathrm{~cm}$ in length). The head (or right side) of the density curves show the expansion of the $\mathrm{HE}$ products into vacuum as a linear region which approaches a 0 density value at about $X=17 \mathrm{~cm}$. There is a discontinuity in the slope of the density curves located at about $x=10.5 \mathrm{~cm}$. Once the HE detonation reaches the end of the $H E$, a rarefaction wave is launched. The rarefaction region begins at the slope discontinuity and continues until the density reaches than of the burned HE (still nearly identical to that of the original HE density). The piston has moved in about $1 \mathrm{~cm}$ by this time. The convergence of the CALE calculations as the resolution is increased (especially evident near regions close to the slope discontinuities) to the analytical solution is shown. The largest discrepancy between the calculated densities and the analytical solution remains at the left boundary of the problem (the piston interface). However, there is also a slight discrepancy in the behavior of the HE products vacuum expansion region shown by the higher resolution (32000-64000 zone blue curves) calculations. 
Density vs. $\mathrm{R}(t=2000)$

(multiple resolutions)

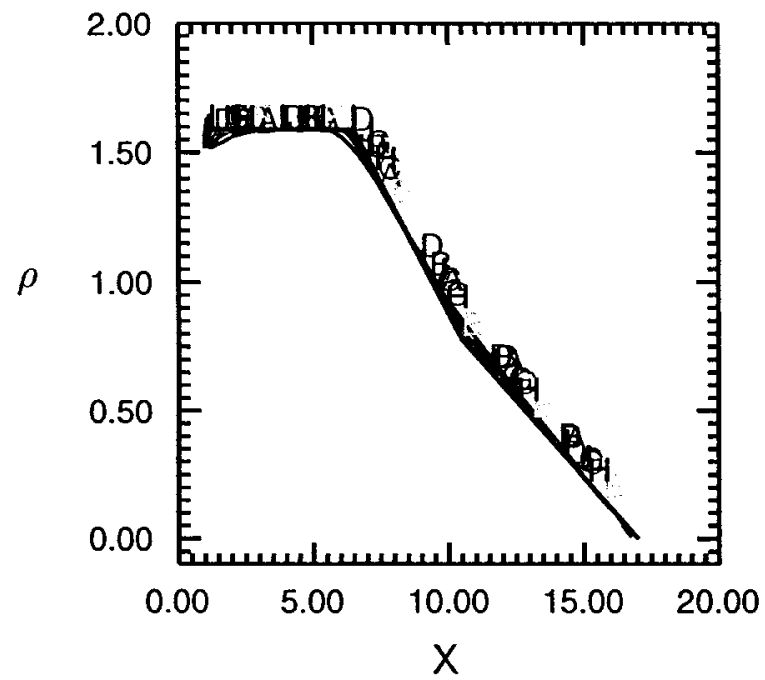

A - I density $[25,50,100,200, \quad 800$,
$1600,3200 \& 6400$ zones]
J density [analytic solution]

Figure 5: Density $(\rho)$ plots from CALE calculations at $t=2000$ for multiple resolutions
Density vs. $\mathrm{R}(t=2000)$ (multiple resolutions [zoomed])

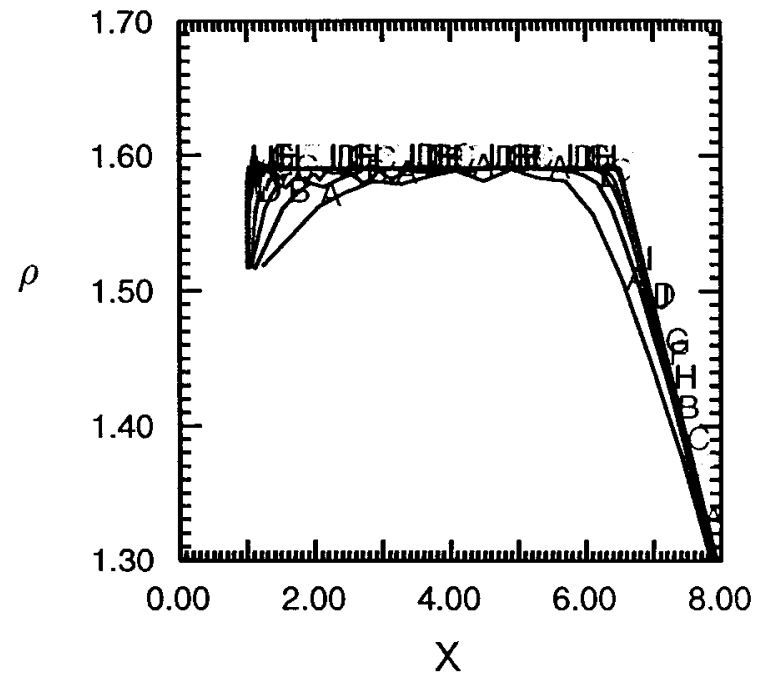

$\begin{aligned} & \text { A - I density } {[25,50,100,200,800,800,} \\ & 1600,3200 \& 6400 \text { zones] } \\ & \mathrm{J} \text { density [analytic solution] }\end{aligned}$

Figure 6: Density $(\rho)$ plots from CALE calculations at $t=2000$ for multiple resolutions [zoomed]

Fig. 6 shows the same data as Fig. 5, but with the plot limits zoomed to show details of the left side of the problem. The convergence of the calculations as mesh resolution is increased is most evident near the two ends of the flat area representing burned, but not yet expanded HE. Beginning just ahead of the piston, especially evident in the lower resolution (25 and 50 zone red curves) calculations, is a a region where the density curves exhibit noise. As the resolution increases, both the width of the boundary interaction region and the amplitude of the noise is reduced.

Curves of density vs. $x$ calculated by the CALE code for resolutions of $25,50,100,200,400$, $800,1600,3200$ and 6400 zones are shown on Fig. 7 taken at the final time $t=7000$. The largest discrepancy between the calculated densities and the analytical solution continues to occur at the left boundary of the problem (piston interface). The density values for the lower resolutions (especially the 25 and 50 zone $A$ and $B$ or red curves) tend to have the largest difference from the analytic density values near the two slope discontinuities $(x \approx 6$ and $x \approx 10)$ and at the piston interface. As the resolution increases, the values of the density appear to be converging towards the analytical solution everywhere except at the piston interface.

Fig. 8 shows the same data as Fig. 7, but with the plot limits zoomed to show details of the left region the calculation. Even at the final time, the $\Delta x$ extent of the boundary interaction region appears to diminish with increasing resolution, but the magnitude of the disturbance remains constant. The noise in the density values, first seen on the $t=2000$ plots, is still present, with the largest noise values occurring near the left boundary of the problem. There is now an observable difference (about $0.3 \%$ ) between the density that the CALE calculations are converging to and the analytic value.

The choice of $\gamma=3.0$ and the velocity of the piston combine to give this problem a simple, linear set of characteristics. These choices allow the analytic solution to be easily calculated. 
Density vs. $\mathrm{R}(t=7000)$

(multiple resolutions)

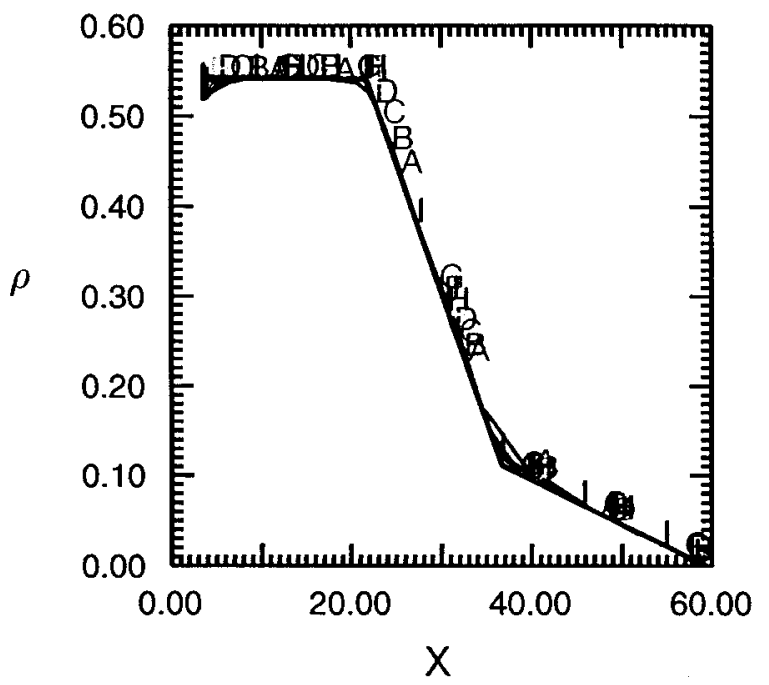

A - I density $[25,50,100,200, \quad, 800$,

$J$ density [analytic solution]

Figure 7: Density $(\rho)$ plots from CALE calculations at $t=7000$ for multiple resolutions
Density vs. $\mathrm{R}(t=7000)$

(multiple resolutions [zoomed])

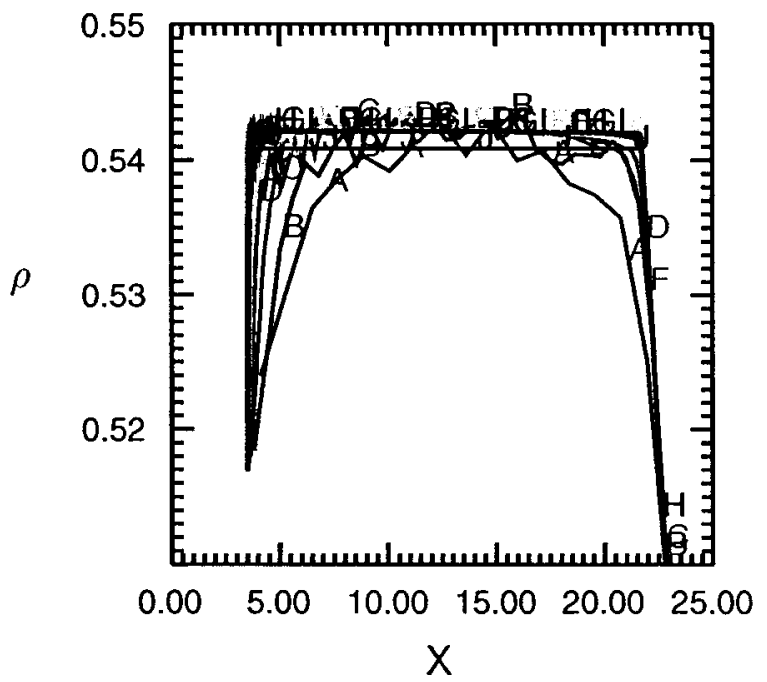

$$
\begin{gathered}
\text { A - I density }[25,50,100,200, \quad 800, \\
1600,3200 \& 6400 \text { zones } \\
\text { J density [analytic solution] }
\end{gathered}
$$

Figure 8: Density $(\rho)$ plots from CALE calculations at $t=7000$ for multiple resolutions [zoomed]

With the exception of the initial rise in density caused by the HE detonation wave - the density of each zone either remains constant or decreases. Since this problem creates no compressions (except at the detonation front which is handled separately), CALE's standard, shock capturing, artificial viscosity $(Q)$ should never be needed. Should the artificial viscosity be turning on anyway this could explain part of the $0.3 \%$ difference between the density of the CALE calculations and the analytic solution at the left edge of the problem. Fig. 9 shows the CALE calculated densities at the final time $t=7000$ using the scalar, monotonic $Q$, the edge based Von Neumann $Q$, and no $Q$ from 800 zone calculations. The scalar, monotonic $Q$ result (curve $A$ or blue line) is identical to that shown previously on Fig. 8. The Von Neumann $Q$ result (curve $B$ or green line) produces values of density greater than the standard case. The linear smoothing coefficient for the Von Neumann $Q$ must be set lower than that of the limited monotonic $Q$, accounting for the increased noise level exhibited by the Von Neumann density curve. The no $Q$ results (curve $C$ or red line) produces density values slightly less than the standard case, closest to that of the analytic solution. However, the noise is large (the displayed curve has been passed through a 5 point filter) indicating that the shock capturing $Q$ algorithm is responsible for significant smoothing of the density. But, even without any $Q$, the CALE calculations are still converging to density values higher than that of the analytical solution.

Curves of pressure vs. $x$ calculated by the CALE code for resolutions of 25, 50, 100, 200, $400,800,1600,3200$ and 6400 zones are shown on Fig. 10 taken at the final time $t=7000$. All sections of the density slice plots (at least for the analytical solution) were linear. The middle region of the pressure solution is nonlinear. This is the part of the rarefaction wave which extends further than the shock produced by the piston. The CALE pressure plots show many of the same features displayed by the density calculated values. The boundary interaction, now visible as an increase in pressure, is the only area where the calculation appears not to be converging to the analytical solution. 
Density vs. $\mathrm{R}(t=7000)$

(Scalar Monotonic Vs. Von Neumann Q's)

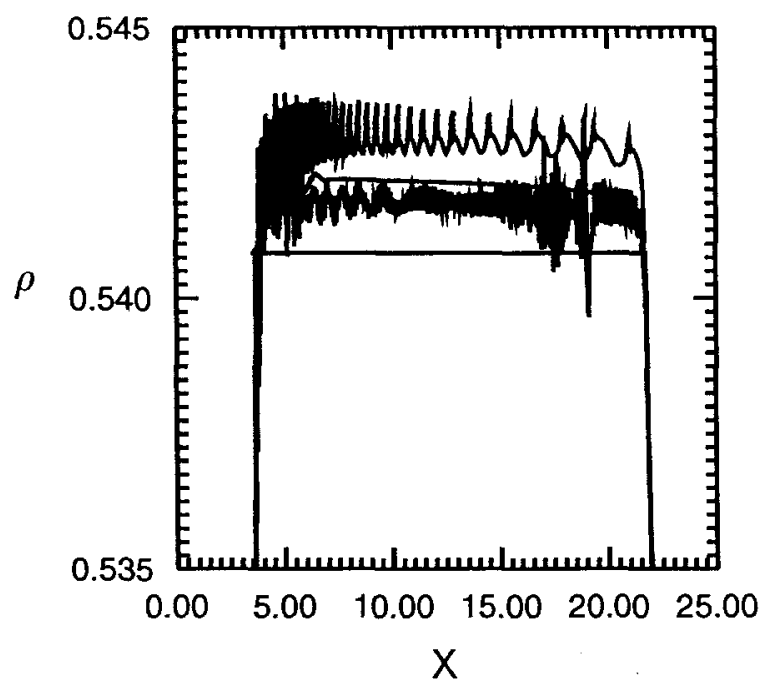

A Scalar Monotonic $Q$ (blue)

$B$ Vector Von Neumann $Q$ (green)

$C$ No $Q$ (red)

D Analytic Aolution (black)

Figure 9: Density $(\rho)$ plots from CALE calculations at $t=7000$ for multiple Q's
Pressure vs. $\mathrm{R}(t=7000)$

(multiple resolutions)

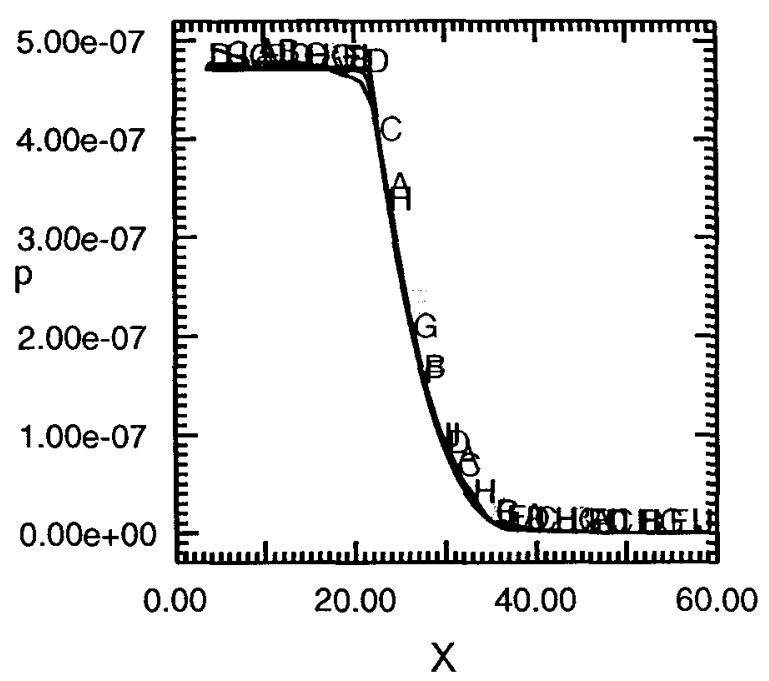

A - I pressure $[25,50,100,200$,
$1600,3200 \& 6400$

$\mathrm{J}$ pressure [analytic solution]

Figure 10: Density $(\rho)$ plots from CALE calculations at $t=7000$ for multiple mesh resolutions

The analytical solution of the velocities evaluated at the final time $t=7000$, shows three linear areas of increasing velocity as the $x$ coordinate increases. The CALE calculated velocities (not shown) very nearly overlay the analytical velocities at all resolutions.

The relative convergence rate (not shown) is obtained by applying Richardson extrapolation for three resolutions on a point by point basis. The result is noisy and requires smoothing and is at times difficult to interpret. The extrapolation process is valid only when the numerical algorithms are converging at the asymptotic limit. For the Heprod problem, averaging the Richardson extrapolation curves for CALE resolutions of 1600,3200, and 6400 zones over the range $5<x<55$, yields a measured average value of the relative convergence rate of 1.08 for these calculations. This is quite close to the $1^{\text {st }}$ order convergence rate expected for this problem.

The absolute convergence rate measures how quickly the numerical calculations approach the analytic solution. We use a linear $\ell_{1}$ measurement of the error. Fig. 11 shows a plot of the log of the $\ell_{1}$ error vs. the log of the initial $\triangle x$ (the mesh begins with a uniform $\triangle x$ zoning) for the CALE calculations of the Escape of HE Products Test Problem. The absolute convergence rate is the slope of these curves. Any two resolutions provide an estimate of the convergence rate. At least three resolutions are required to determine if the calculations are in the asymptotic convergence range. A linear fit over the entire range of the 1-D calculations ( $B$ or blue curve) results in a convergence rate measurement of 0.325 , much less than 1.0. The slope determined by the three lowest resolution points $(25,50$ and 100 zones) is 0.797 , much closer to the expected value of 1.0. Since the CALE calculations are converging to a result that is different than the analytical solution, the slope of the $\ell_{1}$ error curve decreases monotonically as the resolution is increased. The slope shown by the two highest resolution 1-D points ( 3200 and 6400 zones) is only 0.067 . The $\ell_{1}$ error is expected to remain about constant as the resolution is increased beyond the existing 6400 zone calculation. This behavior suggests that as resolutions exceed 100 zones, the $\ell_{1}$ procedure is more sensitive to differences in the numerical and analytic solutions 


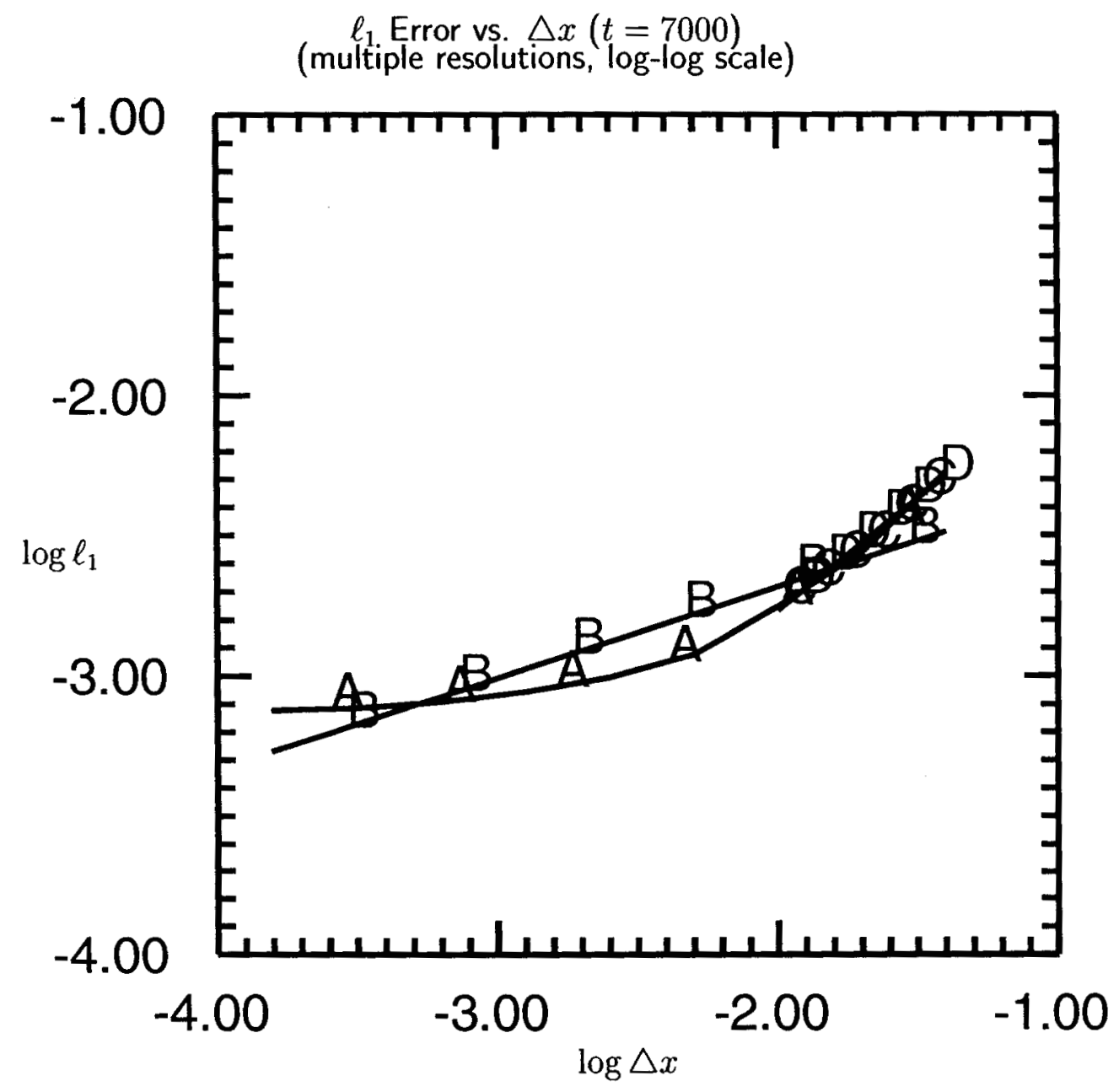

A $\ell_{1}$ norms vs. $\log \triangle x$

B Fit A $1(-2.03+0.325 x)$

$C$ Three lowest resolution points of curve $A$

D Fit C $1(-1.18+0.797 x)$

Figure 11: Absolute $\ell_{1}$ order of convergence for CALE 1-D Heprod calculations

than to the convergence of the numerical solutions. Even though the CALE calculations produce density values that are converging as the mesh resolution is increased, in the limit of small $\triangle x$, CALE will not exactly $(0.3 \%$ error $)$ reproduce the analytic solution. 


\section{Conclusions and Action Items for the Heprod Problem}

A formal analysis of the error behavior, as defined by the $\ell_{1}$ norm of the difference between the analytic and numerical densities, has been completed for the CALE code. These calculations were made using a HE stick only 1 zone wide to represent 1-D behavior from the 2-D CALE implementation. CALE "passes" the viewgraph-norm comparisons with the analytic solution. A relative error Richardson extrapolation for the three highest resolutions suggests that CALE results are converging at about the expected $1^{\text {st }}$ order rate. However, formal analysis of calculations using multiple resolutions shows CALE produces results which diverge from the analytic solution $(0.3 \%$ error). Although there is some dependence of the calculated errors on the code's artificial viscosity (Q), most of the error remains even in the limit of no $Q$. Like the Noh problem, the boundary interaction region near the piston interface is also not eliminated by increasing the resolution of the calculation.

The Heprod problem has uncovered several areas in which more work can help answer some of the questions remaining from this analysis. Some of the identified action items are as follows:

- Many features of the HE burn model seem to be empirically determined. More analysis of many of the options available is warranted.

- The $\ell_{1}$ density error analysis needs to be extended to include pressures, energies (or temperatures), and velocities

- Other codes (ALE3D) need to be analyzed and the analysis needs to be extended to 2 and 3-D implementations

- This analysis needs to be repeated (automated) periodically to monitor changes as simulation codes evolve

The formal analysis of the error behavior of codes, as the resolution is increased, measures effects at levels easily missed by earlier comparisons to the analytic curves at one or two resolutions. 


\section{References}

[1] W. Fickett and W. C. Rivard, "Test Problems for Hydrocodes," LA-5479, August 1974, problem 6, (unpublished). 